\title{
Conjugate deviation of downgaze in the toxic shock syndrome
}

\author{
D.J. Dick ${ }^{1}$, R.D. Harvey ${ }^{1}$, S.J. Hudson ${ }^{2}$ and T.J. Walls ${ }^{1}$ \\ Departments of ${ }^{1}$ Neurology and ${ }^{2}$ Public Health, Newcastle General Hospital, Newcastle upon Tyne, UK.
}

\begin{abstract}
Summary: A patient with the toxic shock syndrome presented with conjugate deviation of downgaze and impaired ocular movement. Focal neurological signs are rare in this syndrome and disordered eye movement has not been previously reported.
\end{abstract}

\section{Introduction}

The toxic shock syndrome (TSS) is an acute illness characterized by fever, headache, confusion, generalized erythroderma, conjunctival hyperaemia, diarrhoea, hypotension and oliguria (Anon., 1980). TSS has been associated with tampon usage in menstruating women and in these cases the vagina is usually colonised by toxin producing strains of Staphylococcus aureus. Although confusion may occur, focal neurological signs are not recognized to be a feature of this syndrome (Shands et al., 1980). We report a patient with the TSS who presented with conjugate deviation of downgaze.

\section{Case report}

A 16 year old Caucasian schoolgirl was admitted with a $2 \mathrm{~d}$ history of malaise, myalgia and a sore throat. On the day of admission she vomited several times and her level of consciousness deteriorated. Her only medication had been single doses of oral ampicillin and prochlorperazine which she had vomited intact.

On examination she was semi-conscious but would localize to pain. She was pyrexial $\left(39^{\circ} \mathrm{C}\right)$, peripherally cyanosed and had striking conjunctival injection (Figure 1). Her blood pressure was $100 / 50 \mathrm{mmHg}$. She was found to be menstruating and an offensive tampon (Super Tampax) was removed. The only focal neurological sign was conjugate spasm of downgaze which was unaffected by the doll's eye manoeuvre or by intravenous procyclidine (Figure 1). Investigations showed neutrophil leucocytosis and slightly raised bilirubin and aspartate transaminase levels, with normal arterial blood gases. An electroencephalogram

Correspondence: D.J. Dick, M.D., M.R.C.P., Regional Neurological Centre, Newcastle General Hospital, Westgate Road, Newcastle upon Tyne NE4 6BE, UK.

Accepted: 30 May 1985 showed a diffuse increase in theta and delta activity but both the cerebrospinal fluid and a computed tomographic scan were normal. Blood cultures were negative but a high vaginal swab grew Staphylococcus aureus of phage type $29 / 52 / 79 / 42 \mathrm{E} / 81 /+$ which was subsequently shown to produce enterotoxin $F$.

Treatment was commenced with flucloxacillin and during the following $48 \mathrm{~h}$ her conditions improved although the spasm of downgaze persisted. After $3 \mathrm{~d}$ the doll's eye manoeuvre could move the eyes to the primary position but not above the horizontal meridian. Four days later her eye movements appeared full but optico-kinetic nystagmus could not be elicited in the vertical plane until $10 \mathrm{~d}$ after her admission. During her clinical recovery she developed a desquamating rash on her hands.

\section{Discussion}

Conjugate deviation of downgaze is an unusual disorder of ocular movement and implies a selective impairment of upward gaze tonus. It always denotes a disturbance of the brainstem and is usually the result of compression of the mid-brain tectum (Plum \& Posner, 1980). However, metabolic disturbances such as hepatic coma or hypoglycaemia and intoxication with sedative drugs may produce a similar clinical picture (Simon, 1978).

The clinical presentation and laboratory investigations leave no doubt that this patient suffered from the toxic shock syndrome. The persistence of the ocular signs and their insensitivity to procyclidine suggests that the transient ingestion of prochlorperazine was not related to their genesis. It was also unlikely to have been due to an anoxic encephalopathy since the arterial $\mathrm{PO}_{2}$ was persistently normal. Although confusion and seizures may occur in the TSS, focal neurological signs are rare (Shands et al., 1980).

C The Fellowship of Postgraduate Medicine, 1985 


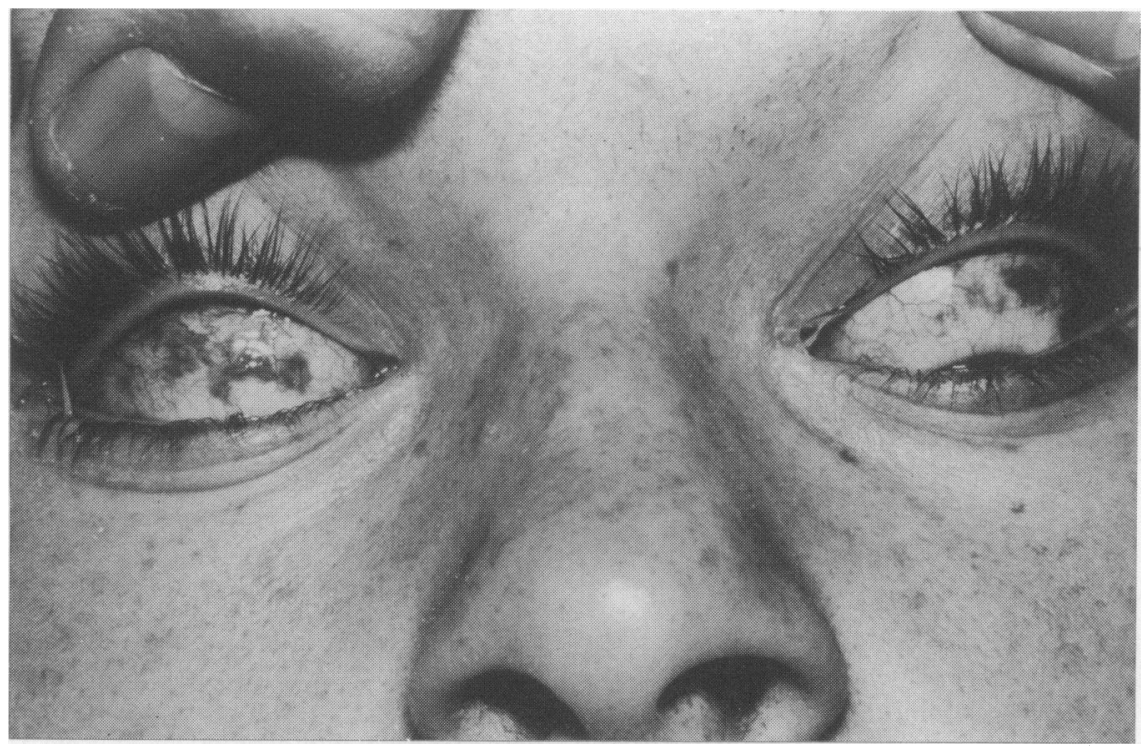

Figure 1 Photograph illustrating conjunctival hyperaemia and conjugate deviation of downgaze.

Disordered vertical gaze has not been reported, and it seems likely that in our patient this was part of the illness itself, although the mechanism by which it occurred is not clear.

\section{References}

ANON. (1980). Toxic shock and tampons. British Medical Journal, 281, 1161.

PLUM, F. \& POSNER, J. (1980). In The Diagnosis of Stupor and Coma. F.A. Davis: Philadephia.

SHANDS, K.N., SCHMID, G.P., DAN, B.B., BLUM, D., GUIDOTTI, R.J., HARGRETT, N.T., ANDERSON, R.L.,

\section{Acknowledgements}

We thank Dr P. Hudgson for his permission to report this 8 case.

HILL, D.L., BROOME, C.V., BAND, J.D. \& FRASER, D.W. (1980). Toxic shock syndrome in menstruating women. New England Journal of Medicine, 303, 1436.

SIMON, R.P. (1978). Forced downward ocular deviation. Archives of Neurology, 35, 456. 